\title{
Time-Dependent Multi-Dimensional Simulation Studies of the Electron Output Scheme for High Power FEL s*
}

\author{
S. J. Hahn ${ }^{\dagger}$, W. M. Fawley, K.-J. Kim, and J. A. Edighoffer \\ Lawrence Berkeley Laboratory, University of California, \\ Berkeley, CA 94720, USA
}

\section{Abstract}

We examine the performance of the so-called electron output scheme recently proposed by the Novosibirsk group[1]. In this scheme, the key role of the FEL oscillator is to induce bunching, while an external undulator, called the radiator, then outcouples the bunched electron beam to optical energy via coherent emission. The level of the intracavity power in the oscillator is kept low by employing a transverse optical klystron (TOK) configuration, thus avoiding excessive thermal loading on the cavity mirrors. Time-dependent effects are important in the operation of the electron output scheme because high gain in the TOK oscillator leads to to sideband instabilities and chaotic behavior. We have carried out an extensive simulation study by using $1 \mathrm{D}$ and $2 \mathrm{D}$ time-dependent codes and find that proper control of the oscillator cavity detuning and cavity loss results in high output bunching with a narrow spectral bandwidth. Large cavity detuning in the oscillator and tapering of the radiator undulator is necessary for the optimum output power.

\section{Introduction}

Recently, the Novosibirsk group[1] has proposed a novel outcoupling scheme, called the electron output scheme, for high power FEL application. In this approach, shown schematically in Fig. 1, the role of the FEL oscillator is to induce the bunching of the electron beams at low optical power, while outcoupling is achieved by extracting the bunched electron beam out of the oscillator via an achromatic transport into a single pass undulator, called the radiator, where it generates intense coherent radiation. The main advantage of the scheme is that the oscillator can be operated at a low intracavity power level by employing a transverse optical klystron(TOK) configuration. This consequently avoids excessive thermal loading on the cavity mirrors. Figure 1 shows a TOK configuration consisting of three undulators and two dispersive sections.

The SELENE proposal[2] employs this scheme with a goal of a high power FEL serving as a ground-based power

*This work was supported at LBL by the Director, Office of High Energy and Nuclear Physics, U.S. Department of Energy, under Contract No. DE-AC03-76SF00098.

tPermanent address: Dept. of Physics, POSTECH, Korea source for several space applications. Previous, timeindependent simulation studies $[3,4]$ of the SELENE proposal indicated that the radiator output power could be as great as 500 times the oscillator intracavity power.

The fact that intracavity power can be made small in an optical klystron oscillator can be understood from the fact that the effective length $L_{e f f}$ of the undulator increases due to the inclusion of the dispersive sections and that the synchrotron oscillation frequency is proportional to the fourth power of the intracavity power. Since an FEL oscillator with low-to-moderate single pass gain reaches saturation when the beam particles execute about one half of synchrotron rotation, one would expect that $P \propto L_{e f f}^{-4}$. On the other hand, the dispersive sections also increase the single pass gain, which lowers the threshold for the onset of the sideband instability. This instability induces significant temporal fluctuations on the outgoing electron beam which should be avoided for the steady-state operation in the final radiator. To counter this instability, one detunes the cavity length and/or increases the single pass cavity loss, which can be treated only in the timedependent approach. Another source of time-dependent phenomena occurs when the pulse length of the electron beam is comparable to the slippage distance in the FEL oscillator (which is increased by the inclusion of the two dispersive sections). Hence, we believe it is important to carry out time-dependent simulations for a reliable evaluation of the electron outcoupling scheme.

In order to explore all these effects plus those due to instantaneous energy spread, emittance, and diffraction (all of which reduce gain), we have carried out 1- and 2-D, timedependent simulations with the FEL1D[6] and GINGER[7] codes. Our goal was temporally stable, low power operation within the oscillator while simultaneously obtaining the high output bunching of the electron beam required for high power radiator performance.

\section{Oscillator Simulation Results}

For our study, we have chosen parameters corresponding to the current Novosibirsk experiment[1]: $I_{b} \sim 10-100 \mathrm{~A}$, $E_{b}=58 \mathrm{MeV}, \lambda_{s}=8 \mu \mathrm{m}, \varepsilon_{n}=20 \pi \mathrm{mm}-\mathrm{mrad}$, and a micropulse duration $\tau_{p}=1000 \lambda_{s} \approx 27 \mathrm{ps}$. The $79-\mathrm{m}$ long optical resonator (corresponding to a minimum micropulse repetition rate of $1.9 \mathrm{MHz}$ ) contains an optical klystron 


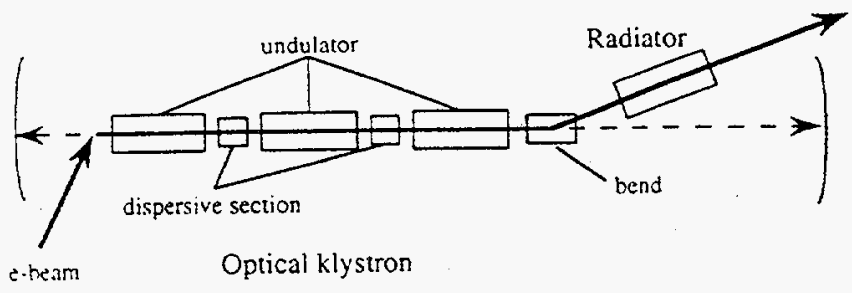

Figure 1: Schematic diagram of the "electron output" FEL system.

consisting of three helical undulators, each with 40 periods of 9 -cm period length $\left(a_{w} \approx 1.2\right)$, separated by two dispersive sections (see Fig. 1).

It is well known[6,5] that cavity detuning and mirror losses play a crucial role for short-pulse FEL oscillators in determining the output spectrum, which can range from a multi-mode chaotic state to a (preferred) single mode stationary state, in between which lies the LCO (limit-cycle oscillation) state[6]. Another important parameter is the strength $D$ of the two dispersive sections. Following Colson, we define $D$ as the ratio $N_{d} / N_{w}$ where $N_{w}(=120$, in the present study) is the total number of undulator periods in the oscillator and $N_{d}$ is the number of undulator periods necessary to produce the drift of each dispersive section. Thus, the effective undulator length is given by $L_{\text {eff }}=(1+2 D) N_{w} \lambda_{w}$.

As mentioned in the previous section, in the low current (i.e. low gain) regime when $D$ and thus $L_{\text {eff }}$ increase, we expect that the intracavity saturation power will decrease. This is confirmed by the results of $1 D$, time-dependent simulation shown in Fig. 2 where the average cavity power (within the micropulse) for three different values of $D$ are plotted versus cavity detuning parameter $-\delta L / \lambda_{s}$. These simulations adopted $I_{b}=8.8 \mathrm{~A}, \delta \gamma / \gamma=0$, and a mode filling factor of 0.11 . We have plotted points corresponding to stationary states only; smaller values of $\delta L$ lead to chaotic or LCO behavior while larger values result in single pass gain below the chosen cavity loss value (10\%). Due to the increase of the single pass gain with larger $D$, the detuning necessary for stationary state behavior also increases with $D$ as is evident from the figure. The average output bunching $b$ which, for the 1D runs, varies inversely with average cavity power, reaching a maximum value of $0.3-0.4$ near the point of FEL threshold. Here $b \equiv\left|<\exp \left(-i \theta_{n}\right)\right\rangle \mid$ where the brackets indicate averaging over the particles's positions in phase space.

Figure 2 also plots the results from $2 D$ time-dependent simulations which include the effects of diffraction, beam emittance, and also instantaneous energy spread $( \pm 0.2 \%)$. The equilibrium e-beam radius $r_{b} \approx 0.6 \mathrm{~mm}$ is $\approx 5$ times smaller than the optical mode radius. The instantaneous energy spread is comparable to $1 / 2 N_{w}^{e f f}$ leading to a reduction of the net gain per pass to about 2.8 for $D=0.2$. This
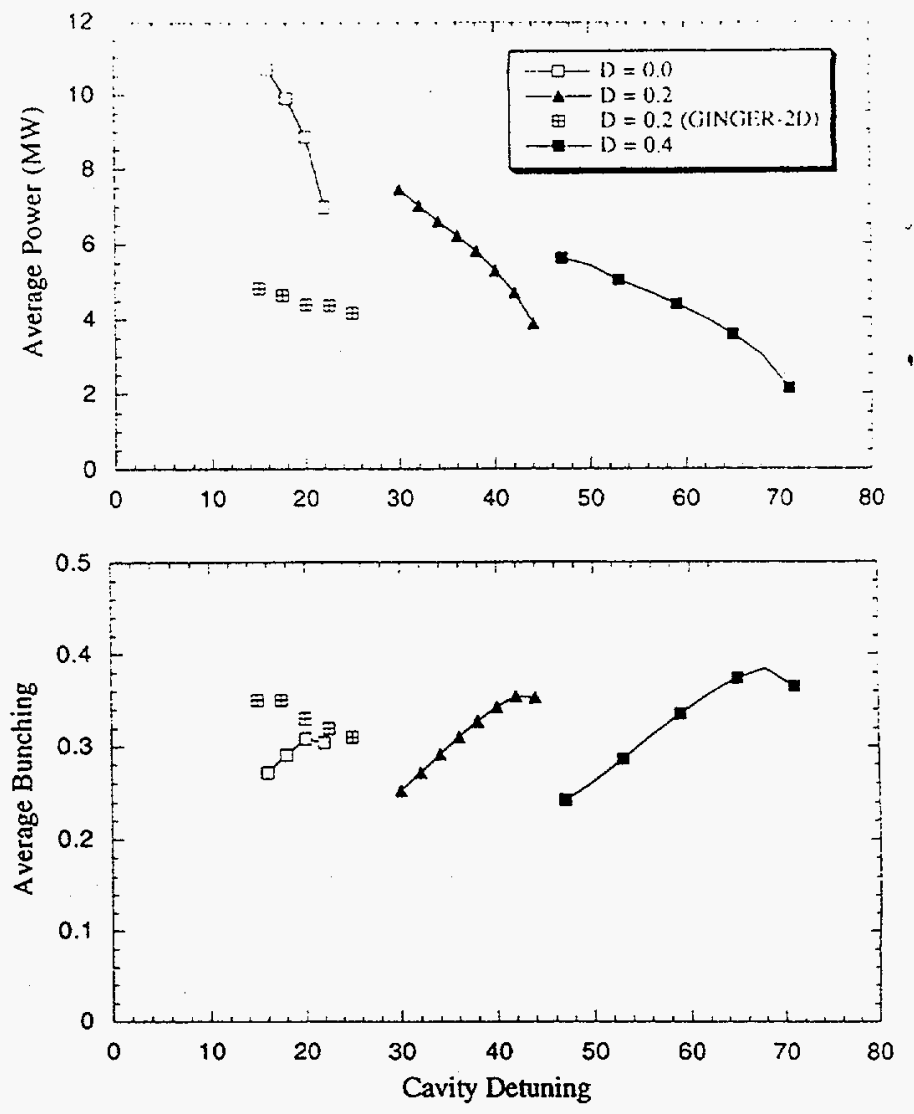

Figure 2: 1D simulation results for the average intracavity laser power and average output e-beam bunching as a function of the cavity detuning for three different optical klystron dispersion strengths $D$ for a low current case $\left(I_{b}=8.8 \mathrm{~A}\right)$. The single pass cavity losses were $10 \%$ for these results. For the case of $D=0.2$, we have also plotted time-dependent, $2 \mathrm{D}$ results that include effects such as diffraction and emittance.

reduction suggests that one must be careful in minimizing the instantaneous energy spread (and output beam emittance if operation at short wavelengths is desired) from the accelerator and transport sections to the undulator. The 2D simulations resulted in stationary states with average output bunching values of $\approx 0.35$ for appropriate values of $\delta L$, which are about a factor of two smaller than for the $1 D$ runs due to the lower gain per pass. When $-\delta L$ is too small (equivalent to too small a cavity loss), the trapped particle instability becomes evident after 30 passes or so.

In the high current (i.e. high gain) regime, the intracavity power and average bunching can show different sensitivity to $D$. For small cavity loss values, as $D$ increases, the average bunching in stationary states decreases as shown in Fig. 3 in contrast to the low gain results. These results were obtained with $I_{b}=88 \mathrm{~A}$, a mode filling fraction of 0.11 , and a $10 \%$ single pass cavity loss. The negative effects produced by the dispersive sections are due to the total phase advance of the particles becoming excessive for large $D$. Another phenomenon that differs in the high 


\section{DISCLAIMER}

This report was prepared as an account of work sponsored by an agency of the United States Government. Neither the United States Government nor any agency thereof, nor any of their employees, make any warranty, express or implied, or assumes any legal liability or responsibility for the accuracy, completeness, or usefulness of any information, apparatus, product, or process disclosed, or represents that its use would not infringe privately owned rights. Reference herein to any specific commercial product, process, or service by trade name, trademark, manufacturer, or otherwise does not necessarily constitute or imply its endorsement, recommendation, or favoring by the United States Government or any agency thereof. The views and opinions of authors expressed herein do not necessarily state or reflect those of the United States Government or any agency thereof. 


\section{DISCLAIMER}

Portions of this document may be illegible in electronic image products. Images are produced from the best available original document. 

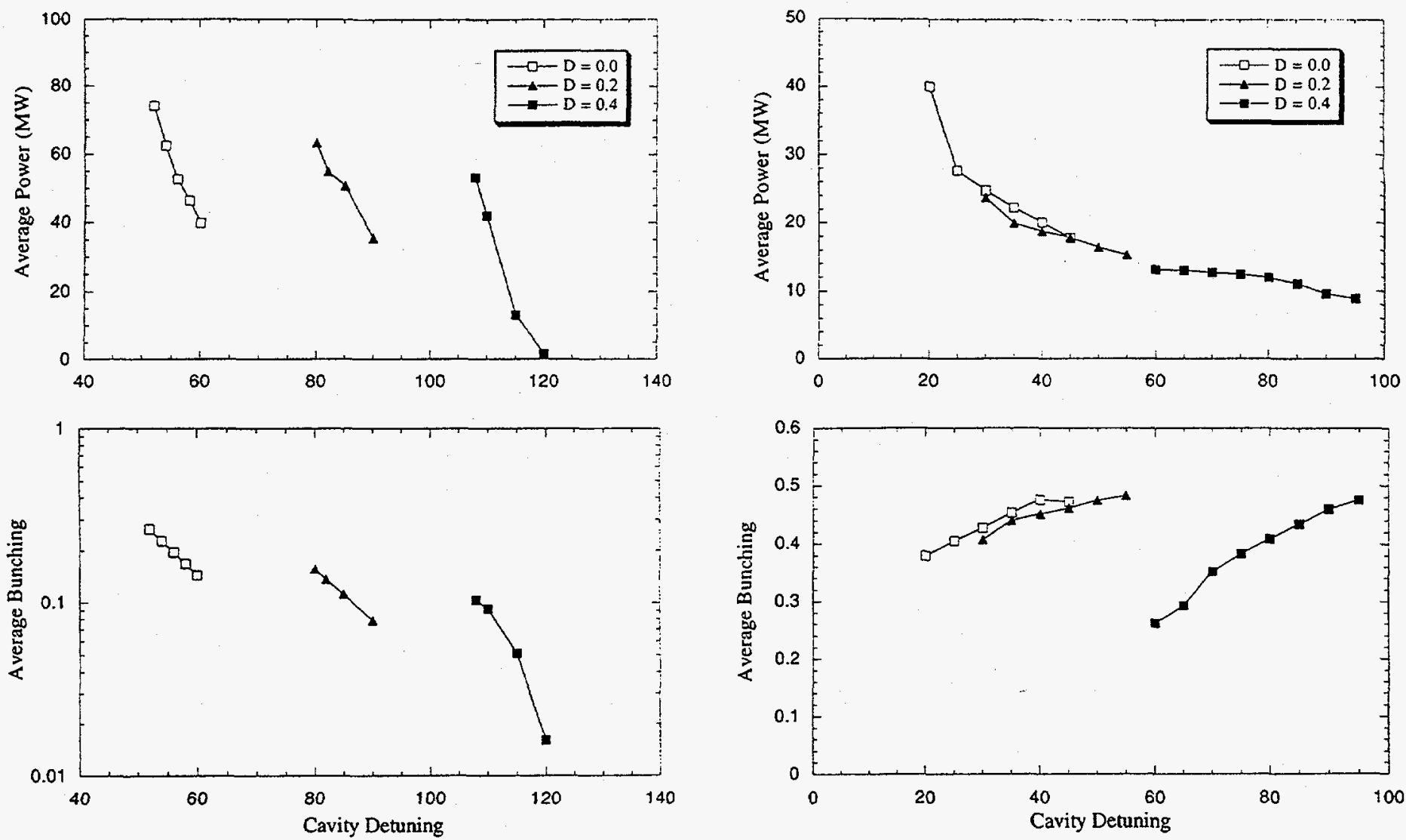

Figure 3: High gain peak intracavity power and output, bunching as a function of the cavity detuning for three different $D$ values for high current $\left(I_{b}=88 \mathrm{~A}\right)$ runs with $10 \%$ single pass cavity loss.

current, high single pass gain regime is that the number of passes necessary to reach nominal power saturation is less than that necessary to reach the single-mode, stationary state. In the low current regime, the situation is reversed. Furthermore, we have noticed that while the start point (in pass number) of the stationary state increases in the low gain regime as the cavity detuning increases, it decreases in the high gain regime.

In order to utilize the advantages of the dispersive sections for high current cases, we found it necessary to increase the single pass cavity losses to $80 \%$. As shown in Fig. 4 , the sensitivity to $D$ and $\delta L$ then becomes quite similar to what was found in the low gain, low current regime. More importantly, the output electron beam is now well bunched for good energy extraction in the radiator undulator.

\section{Radiator Simulation Results}

We modeled the time-dependent radiator performance using as our initial condition the bunched electron beam from the low gain oscillator and a zero amplitude laser signal. Figure 5 shows 1D results for a high current case with $80 \%$

Figure 4: High gain peak intracavity power and output bunching as a function of the cavity detuning for three different $D$ values for high current $\left(I_{b}=88 \mathrm{~A}\right)$ runs. Here the single pass cavity losses were increased to $80 \%$.

cavity losses where $D=0.2$ and $\delta L=-50 \lambda_{s}$ were adjusted to achieve a stationary output state from the oscillator. Both untapered and tapered undulators were considered. For the latter, a simple $5 \%$ linear taper in $a_{w}$ began after $N_{w}=55$ which increased the power by a factor of 5 and strongly suppressed sideband growth and narrowed the output spectrum. The average output power of $156 \mathrm{MW}$ corresponds to an extraction efficiency of $3.0 \%$. These results illustrates the possible advantages of a taper; it may well be possible to increase the output power another factor of two or greater by a more optimal tapering strategy. We also note that an additional dispersive section could be placed between the oscillator and radiator which may further improve the radiator performance.

\section{Conclusion}

In summary, we have the following observations. The electron output scheme works quite well for systems with moderate single pass gain. In this case, one can optimize output bunching and limit intracavity power in the oscillator by judicious choice of cavity detuning and dispersive section st.rengths. The oscillatior then operates in a stationary 

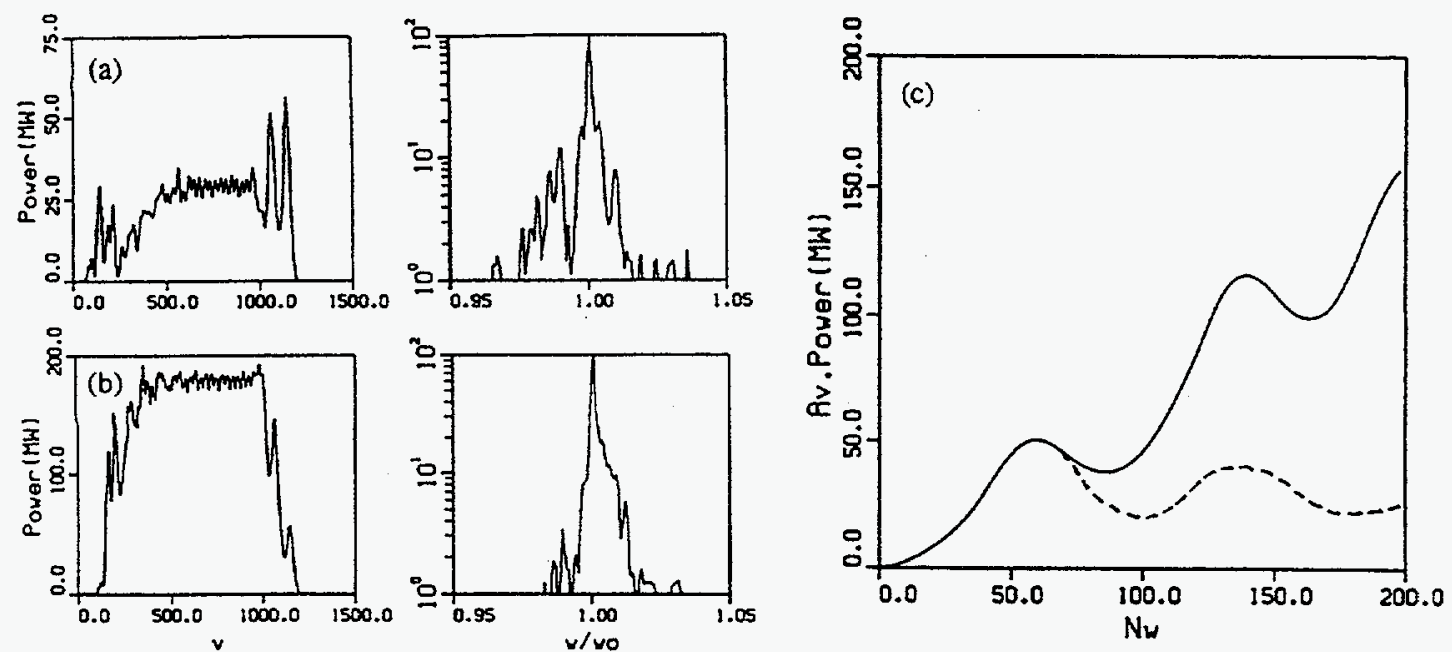

Figure 5: 1D simulation of the radiator for the high current case with $D=0.2, \delta L=-50 \lambda$, and single pass cavity losses of $80 \%$. (a) Optical pulse profile and its spectrum with constant $a_{w}=1.187$ at $N_{w}=200$, (b) tapered case, (c) average power as a function of the undulator period with (solid line) and without (dotted line) tapering.

mode with a narrow spectrum without sidebands and large energy extraction is possible from the radiator. Systems with high intrinsic single pass gain may require additional modifications such as large single pass cavity losses before stable, low power oscillator operation is possible.

\section{References}

[1] G. I. Erg et al., "The project of high power free electron laser using race-track microtron recuperator", Presented at 15th Int. FEL Conf., 1993, Preprint Budker INP 93-75.

[2] H. E. Bennett et al., Nucl. Inst. and Meth., A341, 124 (1994).

[3] D. D. Quick, J. Blau, R. K. Wong, and W. B. Colson, Nucl. Inst. and Meth., A341, ABS 92 (1994);

[4] J. Blau, R. K. Wong, D. D. Quick, W. B. Colson, Nucl. Inst. and Meth., A341, ABS 94 (1994).

[5] See, e.g. R. W. Warren, J. C. Goldstein, and B. E. Newnam, Nucl. Inst. and Meth., A250, 19 (1986). and K. -J. Kim and M. Xie, Nucl. Inst. and Meth., A304, 146 (1991).

[6] D. A. Jaroszynski et al., Nucl. Inst. and Meth., A331, 52 (1993) and S. J. Hahn and J. K. Lee, Phys. Rev. E, 48, 2162 (1993).

[7] R.A. Jong, W.M. Fawley, E.T. Scharlemann, "Modeling and simulation of laser systems", SPIE 1045, 18 (1989). 